\title{
Against Inefficacy Objections: \\ The Real Economic Impact of Individual Consumer Choices on Animal Agriculture
}

\author{
Pre-Publication Draft \\ Published in Food Ethics \\ November $28^{\text {th }}, 2018$
}

\author{
Steven McMullen \\ Associate Professor of Economics \\ Hope College \\ mcmullen@hope.edu \\ 616-395-7579
}

\author{
Matthew C. Halteman \\ Professor of Philosophy \\ Calvin College \\ mch7@calvin.edu \\ 616-526-6726
}

\begin{abstract}
When consumers choose to abstain from purchasing meat, they face some uncertainty about whether their decisions will have an impact on the number of animals raised and killed. Consequentialists have argued that this uncertainty should not dissuade consumers from a vegetarian diet because the "expected" impact, or average impact, will be predictable. Recently, however, critics have argued that the expected marginal impact of a consumer change is likely to be much smaller or more radically unpredictable than previously thought. This objection to the consequentialist case for vegetarianism is known as the "causal inefficacy" (or "causal impotence") objection. In this paper, we argue that the inefficacy objection fails. First, we summarize the contours of the objection and the standard "expected impact" response to it. Second, we examine and rebut two contemporary attempts (by Mark Budolfson and Ted Warfield) to defeat the expected impact reply through alleged demonstrations of the inefficacy of abstaining from meat consumption. Third, we argue that there are good reasons to believe that single individual consumers - not just individual consumers taken as an aggregate - really do make a positive difference when they choose to abstain from meat consumption. Our case rests on three economic observations: (i) animal producers operate in a highly competitive environment, (ii) complex supply chains efficiently communicate some information about product demand, and (iii) consumers of plant-based meat alternatives have positive consumption spillover effects on other consumers.
\end{abstract}




\section{Introduction}

One common argument against meat consumption rests on the belief that consuming meat causes unnecessary animal suffering. Though most consumers do not personally inflict the suffering in question themselves, the thought is that their consumption causes others-particularly modern industrial meat producers - to inflict the suffering on behalf of consumers, responding directly to the demand for animal products that consumer choices create. If this animal suffering is morally significant, and if consumers could avoid causing it by choosing from among many cost- and nutrition-equivalent plant-based alternatives, then a consequentialist may argue that people should stop consuming meat and eat a plant-based diet instead. Peter Singer made this argument famous in publishing Animal Liberation in 1975 (Singer 2009), and it has been a standard part of the intellectual basis for vegetarian and vegan diets ever since. Many critics have challenged this argument, however, by contesting the alleged causal connection between individual acts of meat consumption and animal suffering. If, because of the size and complexity of agricultural markets, purchasing one additional serving of meat does not increase the total amount of future animal suffering, such critics maintain, then there is little reason for the strict consequentialist to abstain.

This objection to Singer's classic articulation of the consequentialist case for vegetarianism is known as the "causal inefficacy" (or "causal impotence") objection. In this paper, we argue that the inefficacy objection fails. We build our case in three steps. First, we briefly summarize the basic contours of the objection and the standard "expected impact" response, and explain why it matters, both in the lives of everyday moral agents and in the philosophical literature. Second, we 
examine and criticize two contemporary attempts to defeat the expected impact reply through alleged demonstrations of causal inefficacy. Third, we argue that there are good reasons to believe that single individual consumers — not just individual consumers taken as an aggregate — really do make a positive difference when they choose to abstain from meat consumption.

Our guiding insight, more specifically, is that the causal inefficacy objection, in each of the versions we consider, is grounded on false assumptions about how the economy operates. Critics observe the size and complexity of agricultural markets and conclude that individual action does not have a unique discernable impact. However, this conclusion simply doesn't follow from any standard models of how these markets actually work, or from the empirical evidence that we have about producer responsiveness in notoriously competitive animal agricultural markets. We argue that an accurate description of how these supply chains function results in considerable certainty about the long run expected (or average) impact of a marginal change in individual consumption habits. We maintain, in summary, that agricultural markets and supply chains are in fact responsive in predictable and often measurable ways to changes in consumer purchasing decisions, and that this responsiveness warrants consumer confidence, contra the causal inefficacy objection, that abstaining from meat consumption does in fact make a positive difference.

Before we proceed, we should clarify two important points about our use of the term "meat consumption" in this context. First, in using the term "consumption," we are concerned to pick out consumer behaviors, i.e., behaviors that engage markets, like purchasing meat directly from a store or restaurant or eating meat that one has paid for indirectly through an institutional meal plan. In the vast majority of cases for individual consumers, meat purchasing goes hand-in-hand with meat eating even though purchasing and eating can and do come apart, as when-for instance-one buys meat for someone else's dinner or one eats meat that one has hunted, scavenged, or received 
as a gift. In this paper, we set aside instances of meat eating (such as freeganism, scavenging, hunting, and the ingestion of gifted foods) that do not involve consumer engagement with markets. Second, in using the term "meat," we take a broad interpretation that includes the vast majority of dairy products and eggs as well, insofar as the markets for these products function in the same ways and raise the same moral concerns about the causation of unnecessary animal suffering. ${ }^{1}$

\section{The Causal Inefficacy Objection and the Expected Impact Response}

The basic idea behind the causal inefficacy objection is that the expected impact of individual consumer choices diminishes as the supply chain becomes longer and more complex. ${ }^{2}$ For example, in a small-scale economy, there is a clear connection between a person's decision to consume meat and the life and death of an animal. A self-sufficient person, raising their own chickens, would kill the chicken only when they intended to consume it. Similarly, in an economy of a few hundred people supported by one or two chicken farmers, if one household stopped purchasing chickens, the farmers could easily see the change in buying habits and adjust their breeding accordingly.

Early critics of Singer's consequentialist argument against animal consumption argued that this simple vision of the economy is not realistic. Frey (1985, chap. 17) and Martin (M. Martin 1976) both point to the fact that, in a large complex market, customers cannot know if their

\footnotetext{
${ }^{1}$ For discussions of moral concerns about eggs and dairy products and their connections to the meat industry, see Jones and Gruen (2015), Hooley and Nobis (2015), and McPherson (2015). ${ }^{2}$ For an accessible overview of the basic idea as articulated by one of this objection's most influential contemporary proponents, see Mark Bryant Budolfson (2015).
} 
purchases have an impact. According to their argument, as the market gets larger, and the distance between consumers and farmers grows, the causal link between consumer choices and animal breeding/slaughter becomes less clear. A consumer's choice to abstain from meat consumption must affect the choices of the grocer, the wholesaler, and the producer, at minimum, before reaching the farmer. As the argument goes, if the purchase of a chicken does not change the purchasing behavior of the grocery store, then the orders down the supply chain will not change, and there will be no impact on animals. In fact, if, at any point in the supply chain, the impact of the consumers' choice is not substantial enough to cause a corresponding reduction in purchases, there will be no impact on animal lives. To these critics, the probability of having an impact on actual animal lives is vanishingly small. If this is the case, then the choice to abstain from meat consumption makes little difference, and the moral motivation for vegetarianism-reducing animal suffering and death - is undercut, at least from within a consequentialist frame of

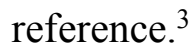

This objection does have a certain intuitive power, and not just over professional ethicists. Who among us has not rationalized that a dubious action we are about to take doesn't really matter in the end? And on the flipside, who among us has not experienced disappointment—maybe even despair and flagging commitment—as we worry that our best efforts to ameliorate some entrenched social problem ultimately come to nothing? In our experience, the causal inefficacy

${ }^{3}$ According to some proponents, not just consequentialist prohibitions of meat consumption are in trouble. Deontological arguments are potentially compromised too insofar as they often employ familiar conceptions of complicity with evil or wrongdoing to explain why abstention from meat consumption is still morally required, as a rule, even though it doesn't discernibly reduce any suffering. See Mark Bryant Budolfson (2017). 
objection is, for many people, a lived-experiential obstacle to considering, much less taking up and sustaining, a commitment to vegetarianism.

The strong intuitive pull of this objection shows up in the philosophical literature in a curious way: while just a few ethicists have attempted to substantiate the alleged causal inefficacy of abstaining from meat consumption as a genuine economic phenomenon, many ethicists simply take it for granted that the objection works (at least for the sake of argument) before moving on to consider other, presumably more compelling arguments against buying and eating meat. This effort to move "beyond difference-making," as Elizabeth Harman describes the strategy, is increasingly common in the recent literature (2015). After declaring the causal inefficacy objection a "serious worry," Harman turns to considering, among other things, whether meat consumption is morally wrong because the consumer participates "as a joint-cause in an act of harming" even though her individual choice is neither necessary nor sufficient to cause the harm in question. Garvey argues that we have an obligation to act in ways that are environmentally-friendly, even if we have no tangible impact (2011). Chignell considers whether purchasing meat can be morally differentiated from opportunistically consuming discarded meat given the industry's purported insensitivity to demand changes (2015). Finally, Martin (2015) develops an account that locates the wrongness of consuming industrially farmed meat in one's complicity in the collective acts of a consumer group "that has the function of signaling demand" for meat "regardless of her actual causal contribution to [these acts]."

The picture that emerges, it seems to us, is that the causal inefficacy objection is ripe for further investigation at the level of the economic assumptions that underlie it. We think there's a fair amount at stake for this investigation, too. After all, we are considering an objection that gets a lot of intuitive traction with people at the ground level, makes detailed empirical claims about 
how agricultural markets work, has proponents who contend that these claims undercut the most influential arguments against meat consumption of the last four decades, and is frequently considered a serious objection by influential philosophers who are moved, at least in part by the force of this objection, to explore other non-difference-making prospects for arguing against meat consumption.

We are sympathetic to the view that there are compelling ways to argue against meat consumption without presuming that individual acts of consumption are difference-making. ${ }^{4}$ Indeed, the development of some of these compelling new arguments has been quite fruitful. However, we think that the consequentialist argument is stronger than the literature suggests, and that a closer look at agricultural supply chains reveals that individual consumer choice still packs a significant causal punch. The real and potentially measurable effects on animal lives that result from our actions should thus remain a central part of how we morally evaluate our choices, even though there are other good arguments against meat consumption.

The standard reply to the causal inefficacy objection is the "expected impact" argument (Matheny 2002; Norcross 2004; Singer 2009; Kagan 2011), according to which any particular chicken purchase may not cause the grocer to increase or decrease the number of chickens that she orders from the wholesaler, but there will be some "threshold chicken" that causes a shift in ordering. For example, a grocery store may choose to order chickens in batches of 10, and thus wait until 10 chickens have been purchased before ordering more. In this way of thinking, the first

\footnotetext{
${ }^{4}$ In an unpublished manuscript, Robert Jones canvasses a variety of these strategies, including those offered by Harman and Martin, and combines them with his own account of "political veganism," arguing that "these solutions can, in concert, answer the challenge [of the causal inefficacy objection].” The paper draws heavily on Gruen and Jones (2015).
} 
nine customers do not have a direct effect, but the tenth customer causes the purchase of 10 chickens when they purchase one. Similarly, any particular grocery store order from the wholesale supplier may not change the number of chickens the wholesaler purchases from the producer, but there will be a threshold at which the wholesaler will alter its buying habits as well.

Each consumer choice thus has a probabilistic impact: there is some probability that their choice will occur on the threshold and have a large effect, and some probability that it will not be on the threshold and have no immediate effect. Because these thresholds are not knowable to the customer, they will have to evaluate the effect of their purchasing in terms of the "expected impact." The expected impact here would mean the probability of being on the threshold times the size of the threshold impact. If chickens are ordered in batches of 50 , and every $50^{\text {th }}$ purchase spurs another order, then the probability of being on a threshold is $1 / 50$ and the impact of a choice at the threshold is 50 chickens. In this case, the expected impact of purchasing a chicken is one chicken death. Adding more layers into the supply chain does not change the nature of the problem, but merely adds more probabilities and effect sizes to calculate. In this threshold setup, lower probabilities will correspond to higher effect sizes, so that the expected impact is usually similar to the impact of a purchase in a less complicated system with less uncertainty. Because the language of the expected impact argument, in terms of thresholds and probabilities, has become standard in the literature, we will use it through much of the paper. In section 3 , however, we will propose a different way of thinking about consumer efficacy.

Frey has criticized the expected impact response, correctly pointing out that it leaves most consumers, in most cases, having no effect, and a few consumers having a large effect (Frey 1985, 214). The effect of any particular consumer action is thus uncertain, and even the probability of having this type of an effect is likely unknowable for most consumers in most situations. That said, 
it is common to push this criticism too far. Frey's ultimate argument lies in the intuition, shared by Martin, that in a large market, the overall effects of consumer action are unknowable. There is a difference, however, between uncertainty regarding the effect of a particular choice, and uncertainty about the effect of such choices on average. To justify any conclusions about the effectiveness of choices overall requires that one say something about how the economy functions, which Frey does not. It is theoretically possible to have an economy that is both large and responsive, or small and unresponsive, depending on the nature of the relationships between the consumer and the supplier.

The two versions of the inefficacy argument that we consider in detail here take up this task of attempting to provide an explanation of how the nature of the economy defeats consequentialist accounts of consumer efficacy:

a. The "Too Insulated" Defeater-Mark Budolfson maintains that it is normal for agricultural production and distribution to include buffers that prevent marginal changes from altering their production decisions. (Budolfson 2018)

b. The "Too Complicated" Defeater-Ted A. Warfield points to the complexity of the economy, arguing that simple stories of cause and effect are impossible (Warfield 2015).

While these arguments are quite different in their particulars, they share a common form. Each posits some characteristic of the economy that ostensibly precludes a predictable market response to a change in the purchasing habits of an individual person. While only Budolfson frames his argument in the terms of this question, both of these authors conclude that individuals do not have a clear moral obligation to abstain from meat consumption. 
Our case against these versions of the inefficacy objection has both critical and constructive components. In section 2 we summarize each version of the objection and critically evaluate the reasons offered for believing that the market is not responsive; in both cases, we deem the reasons unpersuasive. In section 3 we conclude with a separate constructive argument for believing that the market is, in fact, responsive to individual changes in consumer behavior.

\section{2.a. Budolfson: Producer Responsiveness to Changes in Sales}

The heart of arguments about consumer efficacy regarding animal agriculture can be framed as an empirical question: What impact does the purchase of a chicken have on levels of production in the poultry industry? The answer depends on the way in which firms respond to consumer spending. Budolfson makes a multi-part argument against consumer efficacy in these terms. First, he makes a broad claim that consequentialist arguments against purchasing meat are contingent on the assumption that the economic system will translate consumer choices into changes in animal agricultural production. The fact that this moral claim is contingent on economic circumstances means that the consequentialist case is context-specific in an important way that proponents have rarely acknowledged. Second, Budolfson makes the case that in the particular context of industrial animal agriculture in North America, individual meat purchases are very likely not going to have an impact on production--an argument he supports with an account of how these markets function. We agree with the broad premise of Budolfson's important work: claims of consumer causal efficacy are economically contingent. We contend, however, that industrial animal agriculture in North America is highly responsive to consumer decisions, and thus the standard consequentialist arguments against meat eating actually do apply.

To support his empirically-sensitive argument that individual instances of meat consumption are typically non-difference-making in the North American context, Budolfson 
argues that we can observe enough waste and "slack" in the supply chain to know that any given chicken purchase is not near a relevant threshold (Budolfson 2018). If a person is facing a decision with this kind of uncertainty, and they have good information about the probability of being near a threshold, this can dramatically alter the expected impact calculation. Budolfson often considers, by analogy, the question of whether or not a person should vote. In the U.S. system, if a voter knows - from history, news reports, and polling - that a candidate is very likely to win their state by a wide margin, their expected impact from voting is going to be much smaller than a person voting in a close contest. Similarly, if a person knew that their purchase of a chicken was not near the threshold, they could, he argues, purchase the chicken without worry about consequences for animals.

Budolfson is correct in claiming that expected impact calculations cannot always assume that an action, on the margin, would be the same as the average effect of many such actions. The standard expected utility response given by Singer and Kagan can depend crucially on the kind of information that a person has about the location of thresholds. For this reason, it is worth examining the kind of relevant information that Budolfson thinks a meat purchaser can have. The guiding example he develops to illustrate his primary argument is that of "buffers" built into the supply chain for animal products at every level. A certain amount of meat is "wasted" if it is not purchased before the expiration date at the wholesale level and at the supermarket. Excess meat is sometimes sold to producers of pet food or other animal feed. Budolfson concludes:

[T] he crucial issue is not about the magnitude of these buffers, but rather about their reliability: as long as we can know - as we can - that there are sure to be buffers of nontrivial size throughout the supply ... that reduces the probability of a single individual making a difference to a level that quickly becomes nearly infinitesimal, in a way that is analogous to the way that mathematical models explain why the probability of casting a decisive vote in a large-scale election quickly becomes nearly infinitesimal when it is assumed that individuals have very reliable information that one candidate has a nontrivial lead over the others (Budolfson 2018). 
The existence of these "buffers," in general, is not disputable. Building some buffers into a supply chain is the standard way to deal with uncertainty at both sides of a market (Lapide 2008). Buffers are designed as intentional "slack" in the system, so that if consumers decided to purchase more or less than expected, or if production goes slower than expected, there are extra goods available and other uses for goods that remain unsold. However, the fact that food is wasted or held in reserve in these ways simply does not tell the consumer anything about whether she is near a threshold. In fact, it is not clear why the existence of buffers provides any relevant information that makes the situation different from the straightforward cases described by Singer and Kagan. To understand why we will consider two scenarios in turn, each giving different ways these buffers could be related to a production decision threshold.

First, it is possible that some amount of these buffers has to be "used up" or get very large before production will increase or decrease. For example, an egg producer might find that it is cheaper to stock a local warehouse all at once each week, and only restock when the warehouse is almost empty. In this case, consumers would have to purchase a warehouse full of eggs before a new restocking order was made. If this is the case, then the buffers are just functioning as part of the threshold framework described by Singer and Kagan. The number of eggs in a warehouse shipment would be the size of the threshold that needed to be overcome.

Alternatively, it is also possible that some buffer is a constant part of the system, and will not change in response to consumer choices. This is particularly likely with food waste. There is some imprecision in production and shipping that is just too expensive to eliminate. For example, there might be a potential electronic system that would track chicken age, location, and quality, and thus minimize waste, but if that system cost the firm more money than it would save, firms 
might choose to dispose of some chickens rather than track them more precisely. This kind of waste would be present in the system whether demand was high or low, and might exist at all levels of the supply chain. Note that a consumer decision to consume one more or one less chicken will not have any effect on this kind of waste - the waste would be exactly the same regardless of consumer choices. The key question is whether knowledge of the mere existence of such waste provides any information to a consumer. We contend that it does not, unless the consumer knows that a particular animal product has already been discarded. ${ }^{5}$ The mere knowledge that there is waste in the system overall, or even that there is waste at one's particular supermarket, does not give the customer any information about whether their purchase will influence the retailer to order more or fewer products. Similarly, the knowledge that buffers exist does not tell a consumer anything about their location relative to those buffers. It is quite possible that wasted meat in your supermarket gives the consumer information that their producer is near the threshold, since their normal purchasing pattern could have been excessive. The normal case, however, is that consumers know little to nothing about the distance to a threshold at their particular retailer, and even less further down the supply chain.

Note that this situation is very different than the case of a voter who lives in a state dominated by one political party in a winner-take-all presidential race. In the election example, it is quite plausible that a voter would have some good information about the plausibility of their vote making a difference. Some voters would know that their vote was unlikely to change the race outcome, and other voters, in "swing states" would know that their vote was much more likely to make a difference. The chicken consumer, however, does not have analogous information.

\footnotetext{
${ }^{5}$ It is common for consequentialist ethicists to make exceptions for "freegan" or discarded animal products, since these are already outside the system of production.
} 
Because consumers rarely know what is going on in their own grocery store, much less in the longer supply chain, neither those near nor far from the threshold are likely to have this kind of knowledge.

The efficiency of supply chains will be addressed at greater length later in this paper, but it is worth noting that there is good reason to believe that the "buffers" that exist in the system will be as small as possible and will exist independent of the thresholds. Profit margins in agriculture and food retail are quite low (Damodaran 2018). Moreover, animals are bred for very particular markets. This means that when a producer or retailer sells excess unpurchased broilers to a dogfood producer, they are cutting losses. No producer can consistently "waste" products in this manner without going out of business. Stated alternatively, these markets are competitive, and competition between farmers, distributors, and retailers will force these buffers to be as small, and waste as uncommon, as is financially possible. ${ }^{6}$

A second element of Budolfson's argument is that there are many different factors that influence farmers decision-making in addition to prices and purchasing. For example, he argues that the price of feed, the condition of their land, the price of other inputs, the availability of grazing permits, and transportation costs are all more important than the final selling price of the animals in determining herd size for a particular rancher or flock size for a particular chicken grower

\footnotetext{
${ }^{6}$ As evidence for this characterization, note that the movement toward "lean" supply chains, particularly in food production, has focused, particularly on ways to reduce waste in the supply chain (Mena et al. 2014). The increased efficiency of these systems has been well documented, and is the primary cause of the extraordinary concentration of meat production (Azzam 1997; Martinez 1999; Martinez 2002; The Pew Environment Group 2013a), as well as agriculture markets more broadly (Carillo et al. 2016).
} 
(Budolfson 2018). In this he echoes a similar argument made by Nefsky (2011). He notes that most ranchers and growers will raise as many animals as their infrastructure supports, regardless of the price. In economic terms, he is making an argument for inelastic supply at the level of a particular producer.

This characterization of how agricultural markets work is not well grounded. First, it is worth noting that the sheer number of moving variables that impact farmers' decision-making tells us nothing about the impact of consumer purchasing decisions. While there are many variables that affect the profitability of animal agriculture, the profitability of a farm is always jointly determined by costs and revenue, which will always depend on prices. Moreover, the fact that production costs change all the time actually undermines Budolfson's main argument - that consumers can know something about whether they are operating near a threshold. Since the threshold for production decisions is a moving target, determined jointly by prices and costs, it is essentially unknowable for the consumer.

Second, the observation that most farmers will produce as many animals as their land/facilities will sustainably support is exactly what would be expected in a competitive industry. Individual firms, as price-takers, are extremely unresponsive, in the short run, to changes in price. This, however, does not mean that the market is not responsive. When demand for chickens drops, the result is not that a particular farmer chooses to hatch one less chicken. Instead, the thresholdtriggered event is a particular grower's failure to get a contract to raise birds at all, or a delay in the next shipment of birds, a switch to a different type of agriculture, or a rancher's choice to sell her land to a developer. In the very short run, producers can make small changes to their production 
costs and output, ${ }^{7}$ but the real response that matters is the long-run effect, which in this case would be a period of time somewhere between 6 months and a year. Standard economic models of these industries would predict that in the long run, a competitive market will be much more responsive than in the short run.

The important thing to see here is that most suppliers do not change their behavior in response to a price drop, but rather absorb the losses and keep going. The producers that will respond are those on the margin, with the best outside options or the least competitive position in the market. These marginal producers are the ones that will leave the industry in response to lower prices. In a large industry, moreover, competition will always drive the price to the point where the marginal producer is right on the edge of non-participation in the market. ${ }^{8}$ Even in industries

${ }^{7}$ For an example of an agriculture publication describing short-run market responses to changes in final sale prices and feed prices see: Lawrence et al. (2008).

8 A basic description of the model being described here is available in most introductory microeconomics texts in the chapter(s) describing competitive industries, although it would also be a fair description of monopolistically competitive industries, or other models in which there are a large number of suppliers of similar goods Tomek and Kaiser (2014) give this application of the model particularly good treatment. This kind of market is one in which each individual farmer has little to no power over the price of their goods (as with agriculture) and will thus produce the most profitable quantity at the going price. This kind of industry is responsive precisely because of the low profit margins of individual farmers (The Pew Environment Group 2013a). Not only are chicken growers and other producers often making little money per chicken or per hour of labor, they also have an opportunity cost associated with their land, equipment and time that makes the "economic profit" associated with their business even lower than the low reported profits that show 
that are vertically integrated, like the market for chickens, "growers" often operate with heavy debt, barely above poverty, and parent firms give them only short-term contracts (J. MacDonald 2008). A drop in demand can easily leave them without a contract for a season, or with a delay between shipments of birds (The Pew Environment Group 2013b).

It is important to note that this argument does not depend on a frictionless economy with perfect information in which every consumer action results in a price change and a production change. On the contrary, the expected impact argument that comes out of the threshold framework explicitly takes into account the uncertainty attached to the impact of any particular consumer decision. While each consumer choice moves the market toward or away from a particular threshold, the threshold-triggering action is the producer change described here. What is important about this competitive industry argument is that it gives us good reason to believe that producers will be responsive to changes in prices. Indeed, there will be a strong incentive for firms and industries to be as responsive as they possibly can.

The picture that emerges from economic studies of these types of markets is one in which, despite the scale involved, individual consumer actions are reliably, if probabilistically, translated into changes in the quantity supplied to the market. As Budolfson argues, there are ways in which agricultural markets could be organized that would make them resistant to changes in consumer choices. For example, if the government implemented price controls, and promised to buy excess demand from farmers, then farmers could continue producing as demand falls (Lichtenberg and Zilberman 1986). These types of policies have been historically common in agricultural policy, but have been largely abandoned over the last twenty years. Moreover, even under these programs,

up in their annual accounting. In this scenario, a small drop in price or a slower rate of flock deliveries easily hit the tipping point for those farmers on the margin. 
responses to consumer choices were only delayed, since the subsidies acted as a system for smoothing out volatility, rather than expanding the market.

\section{2.b. Warfield: Economic Complexity}

Warfield's argument starts with the premise that the economy is so complex that causal outcomes over long supply chains are unpredictable. Warfield's "phone bill argument," for example, notes that the purchase of meat is similar to paying a phone bill (2015). When you purchase a chicken, some small fraction of your money may support a factory farm that inhumanely raises chickens. Similarly, when you pay your phone bill, if employees at the phone company eat chicken, some small fraction of your money may support a factory farm. In short, he argues that in a complex economy, it is impossible to avoid some economic connection to factory farming. This argument depends on a very different kind of economic uncertainty than does Budolfson's scenario, but the result of the critique for consequentialist arguments about consumer action are similar. The response to both, moreover, depends on an "expected impact" argument, since consumer omniscience is impossible.

The implication is that any argument against meat purchasing must be able to distinguish between the causal connections that tie both the chicken purchase and the phone bill to the industrial farm. Warfield notes that a consequentialist cannot differentiate between these events by examining the intent of the agent, since the morality of the action needs to depend only on the results. More broadly, Warfield calls for a broad consideration of possible impacts. He notes that there could be feedback mechanisms in the economy, such that decreased consumption by one person resulted in increased consumption by another. In particular as one group of people consumes less meat, this could lower the price of meat, thereby encouraging others to consume more (Warfield 2015, 159). The picture he paints is of an economy with so many connections that 
a devoted vegan or vegetarian could not avoid complicity in animal harm, no matter how strict they were about avoiding animal products.

Warfield does not make any strong claims about the economy. His strategy, instead, is to express a general lack of confidence in a person's ability to present any simple causal story. He argues that the complexity of economic interactions requires one of two responses. Either ethicists give up on causal stories about how consumer actions impact production, or else we give reasons why an action like buying meat has a particular set of consequences that are unique in quality or magnitude.

Responding to Warfield requires that we first concede part of his argument: the economy is extremely complex and most economic transactions could, therefore, be connected to animal agriculture. Usually, however, these connections are trivial. Since most purchases result in income for some other person, and most people consume at least some meat from factory farms, economic purity is impossible. This is an important ethical observation. If one's ethical intuition is that a person who buys chicken once a month and a person who buys chicken once each day are morally similar, then Warfield's argument compellingly reminds us that in our economic system, even the most devoted vegan could be in that once-a-month category.

For a consequentialist, however, the magnitude of the impact does matter, and Warfield's argument does not prevent us from making important distinctions. There is good reason to believe, for example, that purchasing a chicken has a much larger impact on the chicken industry than does paying a phone bill. Moreover, paying a phone bill probably has a negligible effect on the chicken industry, similar to that of a toothbrush purchase, or the purchase of any other unrelated good. If we choose to participate in a modern economy, in fact, this kind of second-order involvement with animal agriculture is unavoidable. In light of this, we can rephrase the argument against meat- 
buying in the following way: it is unethical to purchase meat because the expected impact of a meat purchase on animal well-being is worse than any other comparable purchase.

This alternative phrasing of the argument requires making an empirical claim, but importantly, many of the effects that Warfield thinks might be relevant are empirical in nature, and can be measured using standard economic data. In order to respond to Warfield's skepticism, however, we must make a distinction between what is observable on average, and what is observable in a particular case. It is impossible to know, with certainty, whether one is materially supporting an industrial farm when one pays a phone bill. However, we can tell whether, on average, paying a phone bill results in more money for industrial farms. This unpredictability at the individual level should not dissuade us from consequentialist reasoning, for two reasons. First, noise at the level of an individual observation is perfectly consistent with strong causal effects overall. Imagine trying to measure the height of water in a bucket before and after dipping a cup in and removing some of the water. The ripples from the disturbance make any depth measurement at a particular place and time highly uncertain, and the complexity of the wave patterns make each measurement difficult to predict. However, the average outcome will still be easily predictable and causally related to the amount of water removed. Second, the standard "expected impact" argument for abstaining from meat requires only that the average impact is predictable. This means that economic complexity, as long as it conforms to a broader predictable order, is not a challenge to consequentialist moral reasoning.

As a simple example, we will give some evidence that the effect of an unrelated purchase (like a phone bill) is trivial compared to the actual purchase of a chicken. Let us assume that a customer has $\$ 100$ to use as they wish, and are interested in comparing the impact of two different purchases on animal lives. If the money is spent paying a phone bill, about $\$ 75$ will likely end up 
as wages for a worker. ${ }^{9}$ Moreover, in the United States, roughly $2 \%$ of new income is spent on food, ${ }^{10}$ and for every $1 \%$ increase in food expenditures, the average household spends $0.04 \%$ more money on meat and eggs (Alston and Okrent 2012, 20). Given aggregate food consumption statistics, ${ }^{11}$ this means that, on average, $\$ 100$ spent on a phone bill results in close to 1.6 cents more money spent on meat and eggs. ${ }^{12}$ Spending that $\$ 100$ on chicken will have a similar indirect effect, but this effect is negligible. The point is, that even without figuring out the impact of spending on actual production levels, we can estimate that the phone bill expense has a total impact on factory farming that is four orders of magnitude smaller than the same expenditure on chicken.

${ }^{9}$ The share of national income that goes to labor varies over time, between 70 and $85 \%$ (Bivens 2015).

${ }^{10}$ Food consumption expenditures increase only a small amount as people become richer (Regmi and Seale 2010, 11).

${ }^{11}$ The average household income in the U.S. is around $\$ 50,000$, and people in the U.S. spend close to $11 \%$ of their income on food, on average, and $2.88 \%$ on meat and eggs. While these studies are methodologically sound, they are not the most generous numbers in the literature. Even if we search for estimates most damaging to our argument, however, it is difficult to find estimates of new meat spending from a $\$ 100$ increase in income above $\$ 3.45$, leading to the result that buying $\$ 100$ worth of chicken has an effect 28 times bigger than spending that money on other purchases.

12 The relevant calculations: $\frac{0.02 \times \$ 75}{\$ 55000}=0.027 \%$ increase in food consumption, $0.027 \times 0.04=$ $0.00108 \%$ increase in meat and egg spending, and finally, $.0000108 \times \$ 50000 \times 0.0288=$ 1.55 cents of new spending on meat and eggs. 
Clearly, consequentialist considerations should focus on the chicken purchase, and not the phone bill.

What this calculation really indicates, however, is that while the economy is extremely complex, that complexity does not result in uninterpretable chaos. The secondary effects of spending that result from connections between markets only tend to be strong when there is a wellestablished link between the two industries. Thus, when two goods are clearly competitors in the same market, or when two goods are both produced from the same natural resource, their sales and production tend to be related. In most cases, however, we can safely ignore the "phone bill problem" because the relationship between the telephone market and the market for chickens is so weak. Stated positively, it means that we can make moral arguments about the impact of consumer decisions in a meaningful way, even within a complex economy.

\section{Efficient Responsive Supply Chains and Causal Efficacy}

In this final section, we briefly sketch the contours of a positive argument for consumer efficacy. In this section, we are not directly responding to arguments from the authors we engage above, and so there may be some agreement on these points. Our argument has three parts. First, we argue, adopting the "threshold" framework, that there are good economic reasons to believe that long and complex supply chains, in general, will be responsive to consumers. Second, we will argue that the threshold framework can be misleading, particularly when consumer choices are compared to voting. Finally, we will argue that there are at least three positive-feedback mechanisms operating in the economy that make consumer decisions more effective than we might otherwise expect.

First, the responsiveness of agricultural markets can seem counter-intuitive absent an appreciation for the nature of modern supply chains. The long and complicated supply chains that 
connect individual farmers to consumers are actually designed to be more responsive and more reliable than shorter, more local relationships. For example, modern grocery stores have check-out procedures that track the sale of each product and automatically order replacements from the parent companies. Even in industries that are not vertically integrated, standard information technology allows firms to track sales in great detail, down to individual transactions (Salin 2000). In addition, these companies track the rates of orders to optimize shipping and refrigeration times and to minimize waste. Perhaps surprisingly, there is less waste among large centralized distributors than among smaller, more "local" operations because the large distributors are able to serve numerous grocery stores with millions of consumers. In this kind of system, the large distributors that contract with farms actually do know the rate at which chickens are being purchased throughout their network. This efficient supply-chain technology actually should alleviate some causal inefficacy concerns, particularly in those cases when the size and complexity of markets is the impetus for the inefficacy objection. While it is common to assume that smaller, local markets will be more responsive, there is a wealth of evidence that the vertically-integrated agribusiness oligopolies are the most able to respond to individual changes in consumer demand (Albanese 2015).

If this description of the system still seems implausible, note that large food distribution systems have become the norm precisely because of their efficiency (Martinez 1999; Martinez 2002). Even among operating farms, the larger integrated farms have lower costs overall. One recent study found, for example, that largest farms face costs per unit that are $60 \%$ of what the smaller farms face (McBride and Key 2007; J. M. MacDonald and McBride 2009). All of the incentives in the system, as perverse as they may be with regard to animal well-being, push producers to be as responsive as 
is profitable to changes in consumer demand, and to reduce waste. ${ }^{13}$ More responsiveness to consumers, if it can be done at reasonable cost, will only improve profitability. This means that those who argue that the supply chain is not responsive to customers are in the odd position of claiming that these firms are not sensitive enough to the profit motive, which is a difficult position to defend.

Given this description of the way these markets function, we can now describe the causal chain that connects an individual's purchase to a farmer's production decision. When a person decides to stop purchasing chickens, the result is that their local grocery store automatically starts ordering chickens more slowly, to reflect the decreased rate of sale. The distributor (perhaps Chickens R Us) will automatically adjust their shipments of chickens to that store. Since some shipments will require preset bundles of chickens, there will be a threshold at which a delivery of meat comes a day later, to reflect the slower demand. This "threshold" does not mean, however

${ }^{13}$ It will sound odd to some to contend that modern food systems minimize waste. Most of the wastefulness that is observed in food retailers is a function of the scale of these operations, the high cost of labor, and the low cost of individual food items (Bellemare et al. 2017). The push towards lean and responsive supply chains allows firms to be more competitive because they can mandate uniformity in their supply of animals, and increase the flexibility of their supply lines, cutting down on waste (Martinez 1999; Martinez 2002; Taylor 2006; Mena et al. 2014). This counter-intuitive result is closely related to the debate surrounding the environmental sustainability of "local" food markets, where some have argued that "local" is often less sustainable (Mariola 2008; Coley et al. 2009; McWilliams 2010, chap. 1). For the purposes of this paper, it is enough to note that firms have every incentive to order the right amount of food to meet customer demand, and will reduce waste whenever it is profitable to do so. 
that the information going down the supply chain is less precise. As Chickens R Us is managing their supply of chickens in the distribution network, they are also managing the rate at which they send contracts of birds to their "growers" and the number of growers that get contracts. While not every purchase will trigger a production change, there will be some drop in demand that results in a firm hatching one cohort fewer chicks, one shipment fewer chickens, and one less grower contract.

Alternatively, in less integrated markets, the relationship could easily be adjudicated by price changes, where distributors purchase meat, on demand, from auction at the rate they need to keep up with sales which are being closely tracked. A drop in sales will have some probability of dropping the price of beef by one cent, which will influence the auction price of cows, and will influence current herd sizes and the decisions by current ranchers about whether to leave the industry.

Mathematically and conceptually, this still leaves the argument close to where Singer and Kagan started: if there is some probability $(1 / \mathrm{n})$ that any given purchase will occur on a threshold, then the threshold action will trigger a reduction in production of around $\mathrm{n}$ units, yielding an expected impact equal to 1 . Even the argument that lower prices will draw additional consumers is not likely in the long run. Because farmers have no price-setting power over agricultural markets, the price will always be determined by the costs faced by the marginal producers. This means that almost all of the price variability, over the long run, will be determined by production costs, not demand. Correspondingly, there will be a close to 1-to-1 relationship between the purchase of a chicken and the expected impact on production. ${ }^{14}$

14 This argument corresponds to the observation that, over long time periods, supply should be very elastic. As is noted elsewhere (Just 2000; Sumner et al. 2011; Tomek and Kaiser 2014, 56), 
The actual probability of being on a threshold is probably not relevant to the ethical evaluation of meat purchasing, but it can be estimated using some basic knowledge of current industry practice. In the poultry industry, the large "growers" of "broiler" chickens produce, on average, 329,000 chickens per year (The Pew Environment Group 2013b). If the finest adjustment that a chicken distributor can make is to delay a shipment of birds to the grower by one day, then that means the threshold size will be one day's worth of birds for one farm. This number comes out close to 900 birds. As a result, it is likely that a consumer, when choosing to buy a chicken, has close to a 1/900 chance of being on the threshold, and if a consumer decision triggers the threshold event, the impact will be that 900 fewer chickens will be sold that year.

Second, and perhaps most importantly, it seems to us highly misleading to evaluate the moral importance of our consumption decisions as though their probable efficacy in respect to these thresholds is the main measure of their consequences for the supply chain. ${ }^{15}$ While the action in question might be triggered by a consumer choice on the threshold, actions away from the threshold still contribute. This is an important difference between consumer choices and voting.

this long-run elasticity of supply is difficult to measure, and so we don't have accurate industryspecific empirical studies to refer to. Even so, Sumner et. al. point toward good reasons for thinking this is true: (i) over even short periods of time (less than a year) integrators can cancel contracts with growers and (ii) resources used in animal agriculture have many alternative uses. The result is that economies of scale and returns to scale should be constant, and the long-run supply elastic (Sumner et al. 2011). See also the explanations on pages 54-56 and in chapter four of Tomek and Kaiser (2014).

${ }^{15}$ This argument expands on that of Norcross $(2004,233)$. 
Once an election is decided, the votes cease to be effective. With consumer choices, however, a consumer choice has a lasting impact on inventories even if the consumer action was not a threshold-crossing decision. The standard way of thinking about this question assumes a kind of static economy in which a consumer choice either has an immediate impact or no impact. In fact, supply chains are dynamic, meaning that there can be across-time effects that are harder to observe, but just as real. The practice of discussing consumer impact in terms of these thresholds makes the individual contribution to production decisions overly complicated, and puts an arbitrary emphasis on the threshold-crossing decision, when in fact there is a similar contributory impact of those decisions away from the threshold.

To illustrate this point, let's return to the grocer and the chicken purchase. A choice to not buy a chicken might not prevent the grocer from ordering more chickens that day, if the person is not on the threshold. But when the grocer orders, they will still have one additional chicken in inventory, which may impact their next ordering decision, or the one after. This is to say that all consumer actions contribute to some future threshold crossing. In contrast, votes cannot be counted toward future elections.

Finally, it is worth considering feedback-mechanisms in the economy that might mitigate or amplify the impact of a consumer choice. While these do not directly relate to the singledecision impact arguments noted earlier, they contribute to our broader argument that consumers can have confidence that their choices are difference-making. First, there is one obvious mitigating feedback mechanism that has already been mentioned: when consumers purchase less meat, they will drive down the price of meat, which will encourage other customers to buy more. ${ }^{16}$ For reasons described earlier, this is a likely impact in the short run, but over the long-run, because of low

\footnotetext{
${ }^{16}$ Both Budolfson and Martin make this argument in some form.
} 
profit margins for farmers, this cannot have a sustained effect. So while the immediate response to a loss in demand will be lower prices for already-existing meat products, the long-run impact of those lower prices will be lower production (Tomek and Kaiser 2014).

We also suggest that there are likely to be substantial positive feedback mechanisms. In brief, there are at least three reasons to expect an amplified response to the choice to become a vegetarian, which we will call the supply chain effect, the network effect, and the scale effect (McMullen 2016).

The Supply Chain Effect: First, it is worth noting that a decrease in the number of animals killed means that fewer animals are needed for breeding purposes, and in the case of fish, fewer fish are needed as fish food. While the number of such animals varies by industry, one estimate indicated that $16 \%$ of laying hens produced eggs not for consumption but for hatcheries to produce chickens (Harish 2014). This means that eating less meat affects not just the animals directly killed for food, but also the animals exploited and displaced throughout the supply chain. For consequentialists who care about animal lives, this gives additional reason to abstain.

The Network Effect: Additionally, our actions have impacts on other consumers. Each decision to abstain from meat sends a signal to grocery stores and restaurants to provide more vegetarian food options. This in turn makes it easier for other people to make the same choice, because those foods are more readily available. As an example, consider the market for tofu, which is widely available in most markets, but still difficult to find in some areas. Where it is available, the selection is sometimes poor, and the price high. As more people buy tofu, however, it becomes cheaper to distribute and competition pushes down the price. Grocery stores will stock more varieties and place it more prominently in their displays. If there is a critical mass of local vegetarians, restaurants will be sure to include suitable options on their menus. Case studies have 
shown that, producers make choices about marketing and space in response to consumer actions (Moretti 2010).

Moreover, even apart from considerations of marketing and product availability, there is substantial evidence that there are social effects of consumption decisions that will, in aggregate, multiply the effect of individual choices. Social scientists have identified mechanisms through which conspicuous or visible consumption choices influence the choices of peers (Grinblatt et al. 2008). These effects are especially pronounced for choices where consumers are uncertain, and thus make consumption decisions by “following” peers (Banerjee 1992; Bikhchandani et al. 1992). This "network effect" thus refers to all of the ways in which our consumption choices have spillover effects on other consumers. Conformity is economical, and so early vegetarians actually make it easier for others to follow in their footsteps.

The Scale Effect: Finally, closely related to the "network" effect is the impact of economies of scale. Part of the reason that meat is inexpensive is that the production and distribution is done at a large enough scale that costs are kept down. Many meat alternatives, however, have high prices because their customer base is small. Average production costs will decrease as these industries grow, making meat alternatives more competitive with meat. Similarly, innovation efforts have been directed for years toward making meat production efficient, whereas it is only very recently that significant research and development investments have been put into making scalable, sustainable, and delicious plant-based meat alternatives. ${ }^{17}$ This

${ }^{17}$ For more information on innovative companies that are disrupting the traditional landscape of meat production, visit The Good Food Institute online at http://www.gfi.org (accessed on July 1, 2016). For more information on how these companies are raising start-up capital, visit New Crop Capital online at http://www.newcropcapital.com (accessed on July 1, 2016). 
investment is possible only when the market for these alternative foods gets large enough to warrant new product development (McMullen 2016).

Though many may share the intuition that the economy is too large and complex for one consumer to have a real effect on the lives of animals, or that the probability of having an effect is just too small, ${ }^{18}$ we believe that these conclusions are not warranted by the way animal agricultural supply chains actually work. The intuition behind consequentialist inefficacy arguments depends on the impact of an individual action being qualitatively different (not just smaller) than the impact of larger aggregate decisions. We argue that there is a real qualitative continuity between the impact of an individual choice and the impact of aggregate choices. The difference between the individual and the aggregate effect is only a matter of probability and scale. Thus, individual consumers do have a real impact, though the impact associated with any particular purchase will always be uncertain and difficult to observe. It appears then, that there is a real sense in which consumers have a "vote" in favor of a particular system with each dollar that they spend.

Some of the literature we reference attempts to make a broad, generalizable point about collective action problems, or to make the case for collective political action instead of individual action in the marketplace. We find these conversations interesting and important and do not want to imply any kind of preference for or against action in the market instead of other kinds of activism. The aims of this paper are more modest. It is certainly possible to imagine an economic system in which consumers do not have the power or responsibility described here. Moreover, there may be real differences between the case of meat purchases and other problematic choices highlighted in the literature. Nevertheless, the logic of this argument will be of broad interest to

\footnotetext{
18 This seems to be the position espoused by Chignell (2015).
} 
those studying ethical decision-making in the economy, which extends well beyond the area of animal ethics. ${ }^{19}$

Conflict of Interest Statement: On behalf of all authors, the corresponding author states that there is no conflict of interest.

\section{References}

Albanese, A. 2015. Vertical integration contracts in agriculture: fair trade and efficiency of the food chain. In Envisioning a future without food waste and food poverty, 87-94.

Wageningen Academic Publishers. doi:10.3920/978-90-8686-820-9_9.

Alston, Julian M., and Abigail Okrent. 2012. The Demand for Disaggregated Food-Away-FromHome and Food-at-Hope Products in the United States. Economic Research Report ERR-139. Washington D.C.: USDA Economic Research Service.

Azzam, Azzeddine M. 1997. Measuring Market Power and Cost-Efficiency Effects of Industrial Concentration. The Journal of Industrial Economics 45: 377-386.

Banerjee, Abhijit V. 1992. A Simple Model of Herd Behavior. The Quarterly Journal of Economics 107: 797-817. doi:10.2307/2118364.

Bellemare, Marc F., Metin Çakir, Hikaru Hanawa Peterson, Lindsey Novak, and Jeta Rudi. 2017. On the Measurement of Food Waste. American Journal of Agricultural Economics 99: 1148-1158. doi:10.1093/ajae/aax034.

\footnotetext{
${ }^{19}$ We are grateful to Peter Boumgarden, Jeffrey Bloem, Mark Budolfson, Andrew Chignell, Tyler Doggett, Sarah Estelle, Bob Fischer, Dan Hooley, John Lunn, Nathan Nobis, and Tom Smith for helpful feedback on drafts of this paper. We also benefitted from and much appreciate the input of participants at the Oxford Centre for Animal Ethics annual summer school on "The Ethics of Eating Animals," Oxford, UK, July 2016.
} 
Bikhchandani, Sushil, David Hirshleifer, and Ivo Welch. 1992. A Theory of Fads, Fashion, Custom, and Cultural Change as Informational Cascades. Journal of Political Economy 100: 992-1026.

Bivens, Josh. 2015. The decline in labor's share of corporate income since 2000 means $\$ 535$ billion less for workers. Economic Policy Institute.

Budolfson, Mark Bryant. 2015. Is it Wrong to Eat Meat from Factory Farms? If So, Why? In The Moral Complexities of Eating Meat, ed. Bob Fischer and Ben Bramble. Oxford, New York: Oxford University Press.

Budolfson, Mark Bryant. 2017. The Inefficacy Objection to Deontology: What it is, Why it is Important, and the New Type of Deontological Reason Needed to Reply to it. Unpublished Manuscript.

Budolfson, Mark Bryant. 2018. The inefficacy objection to consequentialism and the problem with the expected consequences response. Philosophical Studies. doi:10.1007/s11098018-1087-6.

Carillo, Felicetta, Francesco Caracciolo, and Luigi Cembalo. 2016. Vertical integration in agribusiness. Is it a bargain? Italian Review of Agricultural Economics 71: 39-49-49. doi:10.13128/REA-18624.

Chignell, Andrew. 2015. Can we Really Vote with our Forks? Opportunism and the Threshold Chicken. In Philosophy Comes to Dinner: Arguments About the Ethics of Eating, ed. Andrew Chignell, Terence Cuneo, and Matthew C. Halteman, 182-202. New York: Routledge. 
Coley, David, Mark Howard, and Michael Winter. 2009. Local food, food miles and carbon emissions: A comparison of farm shop and mass distribution approaches. Food Policy 34: 150-155. doi:10.1016/j.foodpol.2008.11.001.

Damodaran, Aswath. 2018. Operating and Net Margins by Sector.

Frey, R. G. 1985. Rights, Killing and Suffering: Moral Vegetarianism and Applied Ethics. Oxford: Blackwell Publishers.

Garvey, James. 2011. Climate Change and Causal Inefficacy: Why Go Green When It Makes No Difference? Royal Institute of Philosophy Supplements 69: 157-174. doi:10.1017/S1358246111000269.

Grinblatt, Mark, Matti Keloharju, and Seppo Ikäheimo. 2008. Social Influence and Consumption: Evidence from the Automobile Purchases of Neighbors. The Review of Economics and Statistics 90: 735-753.

Harish. 2014. The forgotten mothers of the chickens we eat. Counting Animals: A place for people who love animals and numbers.

Harman, Elizabeth. 2015. Eating Meat as a Morally Permissible Moral Mistake. In Philosophy Comes to Dinner: Arguments About the Ethics of Eating, ed. Andrew Chignell, Terence Cuneo, and Matthew C. Halteman. New York: Routledge.

Jones, Robert, and Lori Gruen. 2015. Veganism as an Aspiration. In The Moral Complexities of Eating Meat, ed. Bob Fischer and Ben Bramble. Oxford, New York: Oxford University Press.

Just, Richard E. 2000. Some Guiding Principles for Empirical Production Research in Agriculture. Agricultural and Resource Economics Review 29: 138-158. doi:10.1017/S1068280500005281. 
Kagan, Shelly. 2011. Do I Make a Difference? Philosophy \& Public Affairs 39: 105-141. doi:10.1111/j.1088-4963.2011.01203.x.

Lapide, Larry. 2008. How Buffers Cain Mitigate Risk. Supply Chain Management Review: 6-7. Lawrence, J. D., J. Mintert, J. D. Anderson, and D. P. Anderson. 2008. Feed Grains and Livestock: Impacts on Meat Supplies and Prices. Choices : the magazine of food, farm, and resource issues 23: 11-15.

Lichtenberg, Erik, and David Zilberman. 1986. The Welfare Economics of Price Supports in U.S. Agriculture. The American Economic Review 76: 1135-1141.

MacDonald, James. 2008. The Economic Organization of U.S. Broiler Production. Economic Information Bulletin EIB 38. Washington D.C.: USDA Economic Research Service.

MacDonald, James M., and William D. McBride. 2009. The Transformation of U.S. Livestock Agriculture: Scale, Efficiency, and Risks. Economic Information Bulletin EIB-43. Washington D.C.: U.S. Department of Agriculture.

Mariola, Matthew. 2008. The local industrial complex? Questioning the link between local foods and energy use. Agriculture and Human Values 25: 193-196.

Martin, Adrienne. 2015. Factory Farming and Consumer Complicity. In Philosophy Comes to Dinner: Arguments About the Ethics of Eating, ed. Andrew Chignell, Terence Cuneo, and Matthew C. Halteman. New York: Routledge.

Martin, Michael. 1976. A Critique of Moral Vegetarianism. Reason Papers 3: 13-43.

Martinez, Steve W. 1999. Vertical Coordination in the Pork and Broiler Industries: Implications for Pork and Chicken Products. Agricultural Economic Report AER777. Washington D.C.: USDA Economic Research Service. 
Martinez, Steve W. 2002. Vertical Coordination of Marketing Systems: Lessons From the Poultry, Egg, and Pork Industries. Agricultural Economic Report 807. Washington, DC: U.S. Department of Agriculture.

Matheny, Gaverick. 2002. Expected Utility, Contributory Causation, and Vegetarianism. Journal of Applied Philosophy 19: 293-297. doi:10.1111/1468-5930.00223.

McBride, William D., and Nigel Key. 2007. Characteristics and Production Costs of U.S. Hog Farms, 2004. Economic Information Bulletin 32. Washington D.C.: U.S. Department of Agriculture.

McPherson, Tristam. 2015. Why I Am a Vegan (and You Should Be One Too). In Philosophy Comes to Dinner: Arguments About the Ethics of Eating, ed. Andrew Chignell, Terence Cuneo, and Matthew C. Halteman, 73-91. New York: Routledge.

McMullen, Steven. 2016. Animals and the Economy. London: Palgrave Macmillan.

McWilliams, James E. 2010. Just Food: Where Locavores Get It Wrong and How We Can Truly Eat Responsibly. Reprint edition. New York, NY: Back Bay Books.

Mena, Carlos, Leon A. Terry, Adrian Williams, and Lisa Ellram. 2014. Causes of waste across multi-tier supply networks: Cases in the UK food sector. International Journal of Production Economics 152. Sustainable Food Supply Chain Management: 144-158. doi:10.1016/j.ijpe.2014.03.012.

Moretti, Enrico. 2010. Local Multipliers. The American Economic Review 100: 373-377. Nefsky, Julia. 2011. Consequentialism and the Problem of Collective Harm: A Reply to Kagan. Philosophy \& Public Affairs 39: 364-395. doi:10.1111/j.1088-4963.2012.01209.x. 
Nobis, Nathan, and Dan Hooley. 2015. A Moral Argument for Veganism. In Philosophy Comes to Dinner: Arguments About the Ethics of Eating, ed. Andrew Chignell, Terence Cuneo, and Matthew C. Halteman, 92-108. New York: Routledge.

Norcross, Alastair. 2004. Puppies, Pigs, and People: Eating Meat and Marginal Cases. Philosophical Perspectives 18: 231-245.

Regmi, Anita, and James L. Seale. 2010. Cross-Price Elasticities of Demand Across 114 Countries. Technical Bulletin TB-1925. Washington, D.C.: USDA Economic Research Service.

Salin, Victoria. 2000. Information Technology and Cattle-Beef Supply Chains. American Journal of Agricultural Economics 82. doi:10.1111/0002-9092.00107.

Singer, Peter. 2009. Animal liberation: the definitive classic of the animal movement. New York: Harper Perennial.

Sumner, D. A., H. Gow, D. Hayes, W. Matthews, B. Norwood, J. T. Rosen-Molina, and W. Thurman. 2011. Economic and market issues on the sustainability of egg production in the United States: Analysis of alternative production systems. Poultry Science 90: 241250. doi:10.3382/ps.2010-00822.

Taylor, David H. 2006. Strategic considerations in the development of lean agri-food supply chains: a case study of the UK pork sector. Supply Chain Management: An International Journal 11: 271-280. doi:10.1108/13598540610662185.

The Pew Environment Group. 2013a. Big Chicken: Pollution and Industrial Poultry Production in America. Washington D.C.: The Pew Charitable Trusts.

The Pew Environment Group. 2013b. The Business of Broilers: Hidden Costs of Putting a Chicken on Every Grill. Washington D.C.: The Pew Charitable Trusts. 
Tomek, William G., and Harry M. Kaiser. 2014. Agricultural Product Prices. Ithaca, NY: Cornell University Press.

Warfield, Ted A. 2015. Eating Dead Animals: Meat Eating, Meat Purchasing, and Proving Too Much. In Philosophy Comes to Dinner: Arguments About the Ethics of Eating, ed. Andrew Chignell, Terence Cuneo, and Matthew C. Halteman, 151-162. New York: Routledge. 